\title{
Breast imaging at Chris Hani Baragwanath Academic Hospital: A clinically relevant audit
}

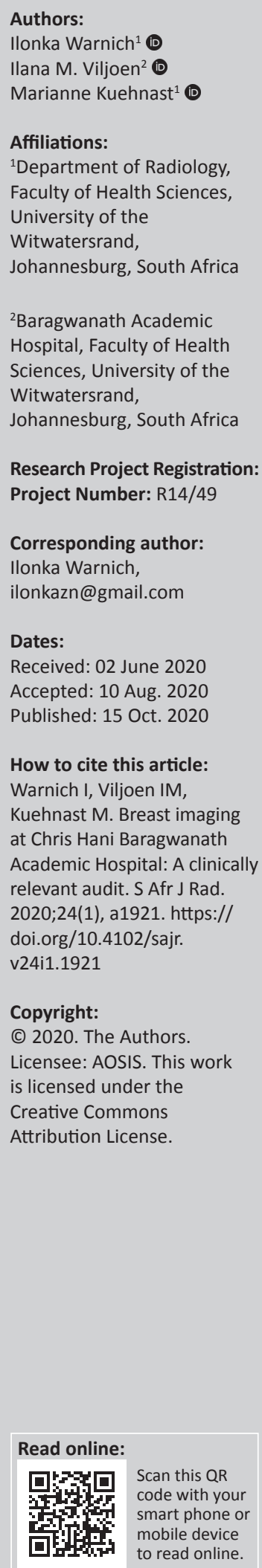

Background: Breast cancer is a major cause of morbidity and mortality worldwide. From experience, we have found that the disease burden at Chris Hani Baragwanath Academic Hospital (CHBAH) is unique with an advanced stage at presentation.

Objective: To perform a breast-imaging audit at $\mathrm{CHBAH}$, focused on interpretive performance and disease burden.

Method: Demographic and imaging data were retrospectively collected over a 6-month period. Data collected and derived followed the audit definitions and rules described within the American College of Radiology-breast-imaging reporting and data system (ACR-BIRADS) atlas (5th edn.). A comparison was made to benchmark values published by the Radiological Society of North America (RSNA).

Results: A total of 1549 mammography examinations were analysed. The screening subgroup $(n=808)$ revealed 11 cancers with a cancer detection rate $(C D R)$ of 13.6 per 1000 studies and a recall rate of 5.94. The diagnostic subgroup $(n=741)$ revealed 130 cancers with a CDR of 175.4 and an abnormal interpretation rate (AIR) of 39 per 100 studies. Along with the positive predictive values, these performance measures for diagnostic mammography were significantly larger than the RSNA-benchmarks $(p<0.0001)$. In addition, the cancer characteristics showed a greater histological mean tumour length, a lower percentage of minimal cancers (defined as ductal carcinoma in situ [DCIS] and invasive cancers $\leq 1 \mathrm{~cm}$ ) and fewer nodal-negative cancers $(p<0.0001)$, in keeping with a more advanced loco-regional stage at presentation.

Conclusion: The study illustrates the challenges faced by a South African breast-imaging unit confronted with advanced loco-regional disease. The cancer burden is highlighted within a community where there is a lack of national screening mammography. The process of performing a basic, clinically relevant audit is simple and should be a routine practice in breast-imaging units.

Keywords: breast cancer; mammography; BI-RADS; breast-imaging audit; diagnostic mammography.

\section{Introduction}

Breast cancer is a major cause of morbidity and mortality worldwide. It is the most common type of female cancer and the leading cause of cancer deaths amongst women. ${ }^{1}$

The need for standardisation in breast imaging has led to the development of the breast-imaging reporting and data system (BI-RADS) by the American College of Radiology (ACR), as summarised in Table $1 .^{2}$ Breast-imaging findings are categorised according to the suspicion of malignancy.

Regardless of standardised reporting systems, there still exists an inter-user variation in the interpretive performance of breast imaging and the threshold to obtain tissue diagnosis. Audits have an essential role in monitoring performance within a facility. ${ }^{3}$

The ACR outlines the basic clinically relevant audit in the 'follow-up and outcome monitoring' chapter within the ACR-BI-RADS atlas (5th edn.). ${ }^{4}$ Annual audits are recommended. The relevance of the audit will be directly proportional to the number of metrics evaluated and should, therefore, be as comprehensive as possible. Separate audits on screening and diagnostic studies are advised, as these show significant statistical differences. ${ }^{4}$ 
TABLE 1: American College of Radiology breast-imaging reporting and data system final assessment categories.

\begin{tabular}{|c|c|c|}
\hline Category & Management & Probability of cancer \\
\hline $\begin{array}{l}\text { 0. Need additional imaging } \\
\text { or prior examinations }\end{array}$ & $\begin{array}{l}\text { Recall for additional imaging } \\
\text { and/or await prior } \\
\text { examination(s) }\end{array}$ & N/A \\
\hline 1. Negative & Routine screening & Essentially $0 \%$ \\
\hline 2. Benign & Routine screening & Essentially $0 \%$ \\
\hline 3. Probably benign & $\begin{array}{l}\text { Short interval follow-up or } \\
\text { continued surveillance } \\
\text { mammography }\end{array}$ & $>0 \%$, but $\leq 2 \%$ \\
\hline 4. Suspicious of malignancy & Tissue diagnosis & $>2 \%$, but $<95 \%$ \\
\hline 4a. Low suspicion & & a. $>2 \%$, but $\leq 10 \%$ \\
\hline 4b. Moderate suspicion & & b. $>10 \%$, but $\leq 50 \%$ \\
\hline 4c. High suspicion & & c. $>50 \%$, but $<95 \%$ \\
\hline $\begin{array}{l}\text { 5. Highly suggestive of } \\
\text { malignancy }\end{array}$ & Tissue diagnosis & $\geq 95 \%$ \\
\hline $\begin{array}{l}\text { 6. Known biopsy-proven } \\
\text { malignancy }\end{array}$ & $\begin{array}{l}\text { Surgical excision when } \\
\text { clinically appropriate }\end{array}$ & $\mathrm{N} / \mathrm{A}$ \\
\hline
\end{tabular}

Source: Sickles EA, D’Orsi CJ. ACR BI-RADS ${ }^{\circledast}$ Follow-up and outcome monitoring. In: ACR BI-RADS ${ }^{\circledast}$ Atlas, Breast imaging reporting and data system. Reston, VA: American College of Radiology, 2013; p. 21-31.

N/A, not applicable.

TABLE 2: American College of Radiology breast-imaging reporting and data system audit definitions.

\begin{tabular}{|c|c|}
\hline Derived data & Definition \\
\hline True positives (TP) & $\begin{array}{l}\text { Positive imaging study with a positive tissue } \\
\text { diagnosis of breast cancer. } \uparrow\end{array}$ \\
\hline False positives (FP) & $\begin{array}{l}\text { Positive imaging study with a negative tissue } \\
\text { diagnosis for breast cancer. }\end{array}$ \\
\hline Positive predictive value (PPV) & $\begin{array}{l}\text { Reflects true positive cases as a proportion of } \\
\text { total positive imaging studies (TP + FP): }\end{array}$ \\
\hline 1. $\mathrm{PPV}_{1}$ & $\begin{array}{l}\text { 1. Based on positive screening cases, with any } \\
\text { result other than routine follow-up (BI-RADS } \\
\text { categories } 0,3,4 \text { and 5). }\end{array}$ \\
\hline 2. $\mathrm{PPV}_{2}$ & $\begin{array}{l}\text { 2. Based on positive examinations with the } \\
\text { recommendation for tissue diagnosis (BI-RADS } \\
4 \text { and 5). }\end{array}$ \\
\hline 3. $\mathrm{PPV}_{3}$ & $\begin{array}{l}\text { 3. Based on positive examinations where tissue } \\
\text { diagnosis was obtained (BI-RADS } 4 \text { and 5). }\end{array}$ \\
\hline Cancer detection rate (CDR) & $\begin{array}{l}\text { Breast cancer-positive cases per } 1000 \\
\text { examinations. }\end{array}$ \\
\hline $\begin{array}{l}\text { Percentage nodal-negative } \\
\text { invasive cancers }\end{array}$ & $\begin{array}{l}\text { Reflected as a percentage of total invasive cancer } \\
\text { cases. }\end{array}$ \\
\hline Percentage 'minimal' cancers & $\begin{array}{l}\text { Defined as invasive cancer } \leq 1 \mathrm{~cm} \text { or ductal } \\
\text { carcinoma in situ (DCIS). Reflected as a percentage } \\
\text { of total cancer cases. }\end{array}$ \\
\hline Percentage stage 0 or 1 cancers & Reflected as a percentage of total cancer cases. \\
\hline \multirow[t]{3}{*}{$\begin{array}{l}\text { Abnormal interpretation rate } \\
\text { (AIR)/Recall rate }\end{array}$} & $\begin{array}{l}\text { Positive assessments, leading to additional } \\
\text { imaging or biopsy, per } 100 \text { examinations: }\end{array}$ \\
\hline & 1. Diagnostic audit: BI-RADS $3,4,5$. \\
\hline & 2. Screening audit (recall rate): BI-RADS $0,3,4,5$. \\
\hline
\end{tabular}

Source: Sickles EA, D'Orsi CJ. ACR BI-RADS ${ }^{\circledR}$ Follow-up and outcome monitoring. In: ACR BI-RADS $^{\oplus}$ Atlas, Breast imaging reporting and data system. Reston, VA: American College of Radiology, 2013; p. 21-31.

BI-RADS, breast-imaging reporting and data system.

$\dagger$, Breast cancer diagnosed within 12 months following the examination; $\ddagger$, No breast cancer diagnosed within 12 months following the examination.

Audit guidelines and definitions are provided within the BIRADS manual (Table 2). Three scenarios for a positive mammogram are described ${ }^{4}$ :

- A screening mammogram leading to anything other than routine follow-up (BI-RADS categories $0,3,4$ and 5).

- A study leading to the recommendation for tissue diagnosis (BI-RADS 4 and 5).

- A study leading to tissue diagnosis being obtained (BIRADS 4 and 5).

True and false positive, as well as positive predictive values (PPVs) can be derived for each of these scenarios $\left(\mathrm{PPV}_{1}, \mathrm{PPV}_{2}\right.$ and $\mathrm{PPV}_{3}$, respectively). The PPV gives the probability that a positive examination accurately indicates the presence of the disease. Of the subcategories, the $\mathrm{PPV}_{2}$ is the most useful within an imaging facility. It is a valuable indicator of interpretive performance, as well as the overall biopsythreshold within the department. The $\mathrm{PPV}_{3}$ reflects clinical practice, and should equal $\mathrm{PPV}_{2}$, where biopsies were performed on all cases in which tissue diagnosis was recommended. ${ }^{5}$

Other valuable performance measures include cancer detection rate (CDR) and abnormal interpretation rate (AIR). Abnormal interpretation rate is referred to as recall rate in screening mammography. ${ }^{4}$

In order for sensitivity and specificity to be derived, negative examinations need to be correlated with a population-based tumour registry to verify the true absence or presence of disease (true- or false-negatives). ${ }^{4}$ No population-based registry is currently available in South Africa. ${ }^{6}$ The data published within the South African national cancer registry lack certain details required for an audit, such as differentiating between screening and diagnostic studies. ${ }^{7}$ It is acceptable to exclude false negatives, sensitivity and specificity from audits, where it cannot be reliably derived. ${ }^{4}$

Metrics evaluating tumour characteristics, such as invasive cancer size, lymph node status and cancer stage are encouraged to be included. The percentage of minimal cancers, node-negative cancers and metastatic cancers can then be derived. The ACR-BI-RADS atlas defines minimal cancers as invasive cancers $\leq 10 \mathrm{~mm}$ or ductal carcinoma in situ (DCIS) of any size. ${ }^{4}$ These metrics give an indication of how early disease is detected, which reflects the major goal of screening and early detection programmes within a country.

The ACR-BI-RADS atlas describes the value of comparing a facility's audit results with acceptable performance parameters. One such value set recommended, is the Radiological Society of North America (RSNA) national performance benchmarks for digital mammography. ${ }^{4}$ Separate publications for diagnostic and screening studies were released in 2017. The data were collected from the breast cancer surveillance consortium (BCSC) and based on the ACR-BI-RADS 5th edition manual. ${ }^{3,8}$

These benchmarks were not intended for use outside the USA, as they reflect the advanced screening programmes and practices specific to the country. ${ }^{5}$ The ACR-BI-RADS manual further describes the limitation of an audit comparison to benchmark data when performed in facilities with relatively small sample sizes, especially a sample obtained from screening-detected cancers. In such cases internal audits or comparison of the facility's trend over time becomes more useful. ${ }^{4}$

Nonetheless, in our opinion, it is of value to analyse deviation from these international benchmarks. Results 
need to be interpreted whilst keeping in mind the vast differences in local practices. This is enough motivation for an incentive to obtain a national mammography database and benchmarks, specific to the South African population and resource-limited setting.

The recent addition of tomosynthesis in screening has assisted radiologists in decreasing recall rates, ${ }^{8}$ and is routinely used as an adjunct to standard digital mammography in many practices. Similarly, the concurrent use of ultrasound during the initial examination, contributing to a combined assessment with mammography, will greatly influence the performance of a unit. ${ }^{4}$ The variable use of these modalities is one of the challenges faced with comparative audits in South Africa, as screening practices are adapted to best suit the population it serves. Immediate reading of screening mammography with the variable addition of ultrasound is a standard practice within many South African breast imaging units. Audit results will differ greatly from facilities where batch reading is performed and patients are subsequently recalled for additional imaging, including tomosynthesis and ultrasound, as commonly done in the USA. ${ }^{9}$

Audit results are also dependent on the screening guidelines within a country. Across the globe there is conflicting data and considerable debate on what these recommendations should entail, particularly in the 40-49 year age group. ${ }^{10}$ The ACR recommends women of average risk for the development of breast cancer to commence annual screening mammography from the age of $40 .{ }^{11}$ The United States Preventative Services Task Force (USPSTF) advises biennial screening mammography within the age group 50-74, with the recommendation that women aged 40-49 can have optional screening after discussion with their healthcare provider. ${ }^{12}$

The South African National Department of Health (NDH) released the Breast Cancer Prevention and Control Policy in $2017,{ }^{6}$ with the major goal of improving breast cancer awareness, early detection and management within the country. Mammography is recognised as the screening method of choice in developed countries, however, South Africa currently lacks the resources to employ and sustain a national screening programme. It is stated that such a programme should only be introduced if it can be ensured that at least $70 \%$ of the target population will benefit from it. ${ }^{6}$ A large percentage of women do not have access to screening mammography, especially those within the rural setting. This contributes to a delay in diagnosis and upstaging of disease. ${ }^{13}$ The NDH recommends clinical breast examination and breast self-examination for early detection of disease. It is, however, recognised that such methods have not yet been proven as efficient screening tools. ${ }^{6}$

The current recommendations by the relevant imaging societies within South Africa are in favour of regular screening mammography. The Radiological Society of South Africa (RSSA) and Breast Imaging Society of South Africa
(BISSA) advise annual screening from the age of 40 , which is in accordance with the recommendation from the ACR. ${ }^{10,11}$

Despite there being no national organised screening programme within the USA, there is a high prevalence of opportunistic screening being performed with a reported $65 \%$ compliance rate (2015). 5,14 Audit results are expected to differ in countries where a lower frequency of screening is done, particularly when evaluating the size and stage of the screen-detected cancers. Earlier detection of tumours is expected when more screening is performed. In addition, because of the lack of surveillance by an organised screening programme, self-funding and the ever increasing risk of malpractice litigation within the USA, the goal of reducing false positive outcomes is deemed less important. This further limits comparative audits with other countries. ${ }^{5}$

Opportunistic screening mammography is also done within South Africa, however, auditing data are generally not kept or available at most facilities. ${ }^{10}$ Therefore, scant research exists on the rate of screening mammography done within the country. The authors suspect the figures to be significantly less than the USA, particularly within the public sector.

There are many countries that do offer national or provincial organised screening programmes, such as Sweden, the Netherlands, Norway, Spain, the United Kingdom, to name a few. Auditing data from these countries are expected to differ. Numerous observational studies from these programmes provide direct proof of the benefit of screening mammography. ${ }^{10,15}$

Chris Hani Baragwanath Academic Hospital (CHBAH) is a major South African tertiary referral institution serving an extensive drainage area. ${ }^{16}$ A relatively large proportion of patients receive diagnostic rather than screening mammography, most of which are referred from the $\mathrm{CHBAH}$ specialist breast clinic. The exception to this is in the month of October, national breast cancer awareness month, ${ }^{17}$ during which screening is promoted.

The relevance of the breast-imaging audit, focused on interpretive performance and disease burden, is to standardise practices, as well as to build a breast-imaging database. The results can be utilised to facilitate quality and skill-improvement methods.

\section{Research methods and design}

A retrospective, descriptive study design was used. The objective was to perform a breast-imaging audit at $\mathrm{CHBAH}$, according to the guidelines outlined in the ACR-BI-RADS 5 th edition manual.

The study population consisted of patients who received a mammogram between 01 June and 31 November 2018. Cases were excluded where inadequate information was available to classify it as diagnostic or screening. Screening studies 
were defined as routine investigations done for asymptomatic patients. Clinical data provided on the radiology report were relied upon to determine these cases. Diagnostic studies included the investigation of breast complaints and the shortterm follow-up of previous abnormal assessments. Additional exclusion criteria were BI-RADS six assessments and previous breast augmentation or mastectomy. In patients with a unilateral mastectomy, imaging of the contralateral breast was included.

The standard imaging protocol included digital tomosynthesis mammography (Hologic Selenia Dimensions with AWS 8000, Laurel Bridge Software). Two-dimensional images (craniocaudal and mediolateral-oblique views) were created from breast tomosynthesis using C-view software. The mammography imaging protocol was the same for screening and diagnostic investigations. Breast ultrasound (Aloka ProSound Alpha 10 system, Version 8 Software) was routinely performed, with the occasional exception of patients presenting with low density breasts (ACR-BI-RADS categories A or B) and unchanged follow-up screening mammography $(n=156)$. When indicated, tissue diagnosis was obtained within the mammography unit. This included ultrasound-guided fine-needle-aspiration, core needle and stereotactic biopsies.

Data were collected from the hospital picture archiving and communication system (PACS - AGFA IMPAX 6.5.1.501). Consultant-approved mammography reports were used to obtain the clinical demographics, indications and relevant imaging findings (breast composition and ACR-BI-RADS final assessments). In cases where each breast was assigned a separate BI-RADS, the highest category was used.

Histology results were tracked for all positive studies (BIRADS 4,5), as well as any other tissue sampling. Results were obtained from the National Health Laboratory Service (NHLS), using the NHLS LABTRAK web-result viewer. Where available, the post-surgical tumour size and nodal involvement were recorded, as stated on the pathology report. These histopathologically proven data were used in the calculation of cancer characteristics. In the presence of multifocal or multicentric disease, the greatest diameter of the largest tumour focus was used as the tumour size.

Data were captured by the primary researcher using Microsoft Excel and the ACR audit definitions and rules were followed (Table 2). It was similar to the methodology used to obtain the RSNA-benchmarks. Derived data were based on mammography examinations with tomosynthesis and additional ultrasound, when performed. Recall rate was defined as any screening investigation with an assessment of BI-RADS 0, 3, 4 or 5. The false-negative values, sensitivity, specificity and presence of metastatic disease were not included in the audit.

Data were analysed using SAS Version 9.2. Descriptive statistics were calculated: numerical data using means with standard-deviations or medians with interquartile ranges (IQRs) and categorical data using frequencies and percentages. The following analytical statistics were used to compare the sample statistics with the published benchmarks: the single proportion binomial test to compare the sample proportion with a proportion in the published benchmarks; the onesample $t$-test to compare the sample-mean with a mean in the published benchmarks; the one-sample Wilcoxon-signedrank test to compare the sample-median with a median in the published benchmarks. The Shapiro-Wilk test was used to investigate if the numerical variables were normally distributed. A significance level of $p<0.05$ was used.

\section{Ethical consideration}

Ethical approval was obtained from the Human Research Ethics Committee, University of the Witwatersrand. Clearance certificate number: M190458.

\section{Results}

A total of 1549 mammography examinations were included in the audit, consisting of $808(52.16 \%)$ screening and 741 (47.84\%) diagnostic studies. Table 3 shows the demographic and breast-imaging data collected and the cancers detected within the various subgroups. The vast majority (79.66\%) of the patients had predominantly fatty or scattered fibroglandular density breast composition (ACR-BI-RADS category A or B). The breast-density distribution showed no significant difference amongst the cancer-positive cases, compared with the non-cancer cases (screening: $p=0.4114$, diagnostic: $p=0.0877$ ).

The majority of the final assessments were BI-RADS 1 or 2 (regarded as negative examinations), within both the screening (93.94\%) and the diagnostic (58.17\%) subgroups.

Abnormal interpretations included BI-RADS categories 3, 4 and 5. The distribution of cancers detected within each of these subcategories is illustrated in Figure 1. The majority of abnormal interpretations and cancers detected were from the diagnostic studies.

Further analysis was done separately for screening and diagnostic studies.

\section{Screening}

The patients were all female and $94.18 \%$ were over the age of 40 years. The median age screened was 56 (interquartile range [IQR] 48.0-64.0), ranging from 23 to 91 years. A known history of previously treated breast cancer was seen in $34.28 \%$ $(n=277)$. The median age of cancer-positive cases was 55 (IQR 47.0-62.0), with a minimum of 39 and a maximum of 71 years (Table 3 ).

The majority of patients underwent the standard mammography imaging protocol with digital breast tomosynthesis and additional ultrasound $(n=652,80.67 \%)$. 
TABLE 3: Audit results for 1549 mammography examinations.

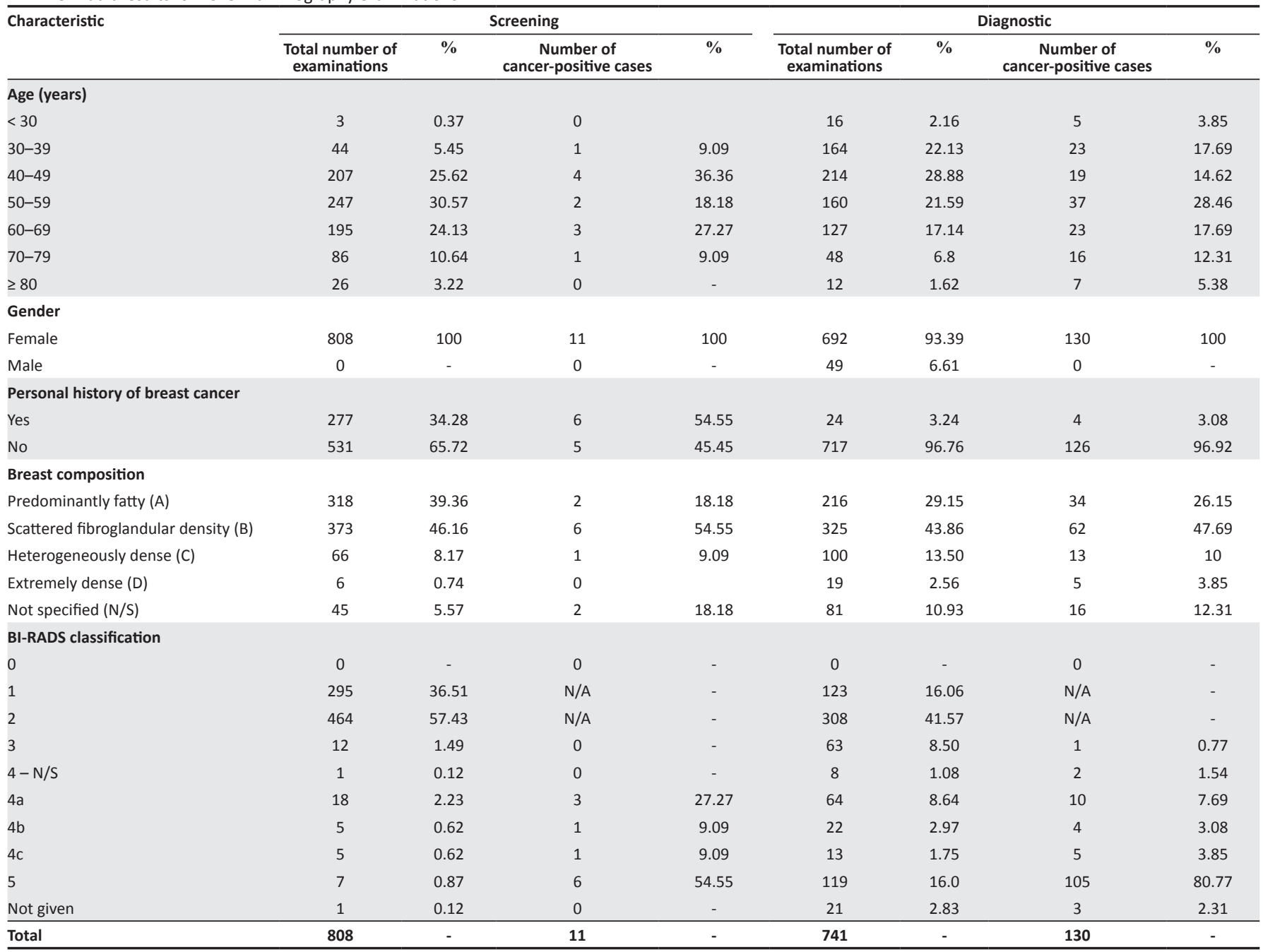

BI-RADS, breast-imaging reporting and data system; N/A, not applicable.

Note: Percentages are based on the total examinations within each column (total values are reported in the last row).

Note: N/A is the number of cancer-positive cases that were not assessed for BI-RADS 1 and 2.

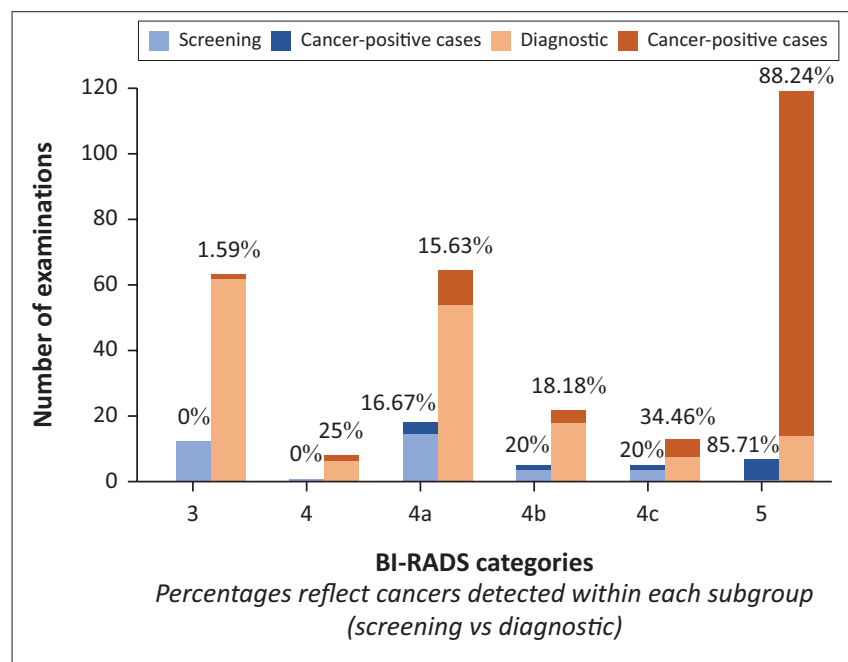

BI-RADS, breast-imaging reporting and data system

FIGURE 1: Breast-imaging reporting and data system categories and cancers detected for abnormal interpretations in screening and diagnostic mammography.

The remaining studies consisted of mammography with tomosynthesis only $(n=156,19.31 \%)$, and represented patients who came for follow-up screening mammography with low density breast parenchyma (ACR-BI-RADS categories A or B) and unchanged negative assessments (BIRADS 1 or 2).

Abnormal interpretations (BI-RADS 0, 3, 4 or 5) were reported in 48 of the 808 screening studies, resulting in a recall rate/ AIR of 5.94 (Table 4$)^{8}$. These patients were all evaluated using mammography, tomosynthesis and ultrasound and given a final assessment. No patients were categorised as BI-RADS 0. Positive interpretations (BI-RADS 4 or 5) constituted 36 studies. Amongst these, tissue diagnosis was obtained in 32 cases $(88.89 \%)$. Eleven cancer cases were diagnosed after a positive screening mammogram, with a CDR of 13.6 per 1000 studies. The $\mathrm{PPV}_{1}, \mathrm{PPV}_{2}$ and $\mathrm{PPV}_{3}$ were 22.92, 30.56 and 34.38, respectively.

There were nine invasive cancers and two DCIS lesions (Table 5) ${ }^{8}$. The invasive cancer cases with a known pathological tumour size $(n=5)$ and nodal status $(n=5)$ demonstrated the following cancer characteristics (Table 5): median tumour length of $20.00 \mathrm{~mm}$ (IQR 9.5-26), minimal- 
TABLE 4: Derived performance measures for screening mammography $(n=808)$.

\begin{tabular}{|c|c|c|c|c|c|}
\hline Measure & $\begin{array}{l}\text { CHBAH } \\
\text { audit value }\end{array}$ & $95 \% \mathrm{Cl}$ & $\begin{array}{c}\text { RSNA- } \\
\text { benchmark } \\
\text { value }\end{array}$ & $95 \% \mathrm{Cl}$ & $p$ \\
\hline $\begin{array}{l}\text { Recall rate (per } 100 \\
\text { studies) }\end{array}$ & 5.94 & $4.51,7.79$ & 11.6 & $11.5,11.6$ & $<0.0001$ \\
\hline $\begin{array}{l}\text { Number of abnormal } \\
\text { interpretations }\end{array}$ & 48 & - & 194668 & - & - \\
\hline $\begin{array}{l}\text { Total number of } \\
\text { examinations }\end{array}$ & 808 & - & 1682504 & - & - \\
\hline $\begin{array}{l}\text { Cancer detection rate } \\
\text { (per } 1000 \text { studies) }\end{array}$ & 13.6 & $7.6,24.2$ & 5.1 & - & 0.0006 \\
\hline $\begin{array}{l}\text { Number of cancers } \\
\text { detected }\end{array}$ & 11 & - & 8529 & - & - \\
\hline $\begin{array}{l}\text { Total number of } \\
\text { examinations }\end{array}$ & 808 & - & 1682504 & - & - \\
\hline $\mathrm{PPV}_{1}$ & 22.92 & $13.31,36.54$ & 4.4 & $4.3,4.5$ & $<0.0001$ \\
\hline $\begin{array}{l}\text { Number of cancers } \\
\text { detected }\end{array}$ & 11 & - & 8529 & - & - \\
\hline BI-RADS $0,3,4,5$ & 48 & - & 194668 & - & - \\
\hline $\mathrm{PPV}_{2}$ & 30.56 & $18.01,46.86$ & 25.6 & $25.1,26.1$ & 0.4976 \\
\hline $\begin{array}{l}\text { Number of cancers } \\
\text { detected }\end{array}$ & 11 & - & 7376 & - & - \\
\hline BI-RADS 4, 5 & 36 & - & 28785 & - & - \\
\hline $\mathrm{PPV}_{3}$ & 34.38 & $20.41,51.69$ & 28.6 & $28.0,29.3$ & 0.4708 \\
\hline $\begin{array}{l}\text { Number of cancers } \\
\text { detected }\end{array}$ & 11 & - & 5945 & - & - \\
\hline $\begin{array}{l}\text { BI-RADS } 4,5 \text { with } \\
\text { biopsy }\end{array}$ & 32 & - & 20763 & - & - \\
\hline
\end{tabular}

Source: Lehman C, Arao R, Sprague B, et al. National performance benchmarks for modern screening digital mammography: Update from the breast cancer surveillance consortium. Radiology. 2017;283(1):49-58. https://doi.org/10.1148/radiol.2016161174

$\mathrm{Cl}$, confidence interval; CHBAH, Chris Hani Baragwanath Academic Hospital; RSNA, Radiological Society of North America; PPV, positive predictive value; BI-RADS, breastimaging reporting and data system.

cancer rate of $42.9 \%(n=3)$ and nodal-negative cancer rate of $60 \%(n=3)$. There were no synchronous bilateral, multicentric or multifocal cancers detected.

The positive cancer cases within the subgroup of women who had a known history of previously treated breast cancer included the following: loco-regional invasive cancer recurrence after previous breast conserving therapy ( $n=2$; known tumour size $20 \mathrm{~mm}[n=1]$ ); invasive cancer involving the contralateral breast $(n=4$; known tumour size $12 \mathrm{~mm}[n=1])$. These patients were all asymptomatic and presented for surveillance screening mammography.

The subgroup of patients with no history of previously treated breast cancer $(n=5)$ showed the following cancer characteristics: CDR of 9.42 (95\% CI [4, 21.8]), invasive cancer median tumour length of $20 \mathrm{~mm}(n=3)$, two DCIS lesions of intermediate- $(n=1 ; 100 \mathrm{~mm})$ and high-grade ( $n=1$; unknown size). The age distribution of screendetected cancers within this subgroup of women were as follows (years): 40-49 $(n=3,60 \%), 50-59(n=2,40 \%)$, median age of 48 (IQR 45-55.5). These index cancer diagnoses were all screened during the months of October (national breast cancer awareness month) and the beginning of November. Three of these patients represented baseline screening studies, whilst the remaining two patients presented for follow-up screening.
TABLE 5: Derived cancer characteristics for screening mammography $(n=11)$.

\begin{tabular}{|c|c|c|c|c|c|}
\hline Characteristic & $\begin{array}{c}\text { CHBAH } \\
\text { audit value }\end{array}$ & $\%$ & $\begin{array}{c}\text { RSNA- } \\
\text { benchmark } \\
\text { value }\end{array}$ & $\%$ & $p$ \\
\hline Cancer type & - & - & - & - & 0.5202 \\
\hline Ductal carcinoma in situ (DCIS) $\dagger$ & 2 & 18.2 & 2644 & 31.0 & - \\
\hline Low grade & 0 & - & Unknown & - & - \\
\hline Intermediate grade & 1 & - & Unknown & - & - \\
\hline High grade & 1 & - & Unknown & - & - \\
\hline Invasive & 9 & 81.8 & 5885 & 69.0 & - \\
\hline Invasive cancer size $(\mathrm{mm})$ : & & & & & 0.5782 \\
\hline $1-5$ & 0 & - & 727 & 12.7 & - \\
\hline $6-10$ & 1 & 20 & 1461 & 25.6 & - \\
\hline $11-15$ & 1 & 20 & 1459 & 25.5 & - \\
\hline $16-20$ & 2 & 40 & 840 & 14.7 & - \\
\hline$>20$ & 1 & 20 & 1228 & 21.5 & - \\
\hline Unknown & 4 & - & 170 & - & - \\
\hline Minimal cancer§ & - & - & - & - & 0.4658 \\
\hline Yes & 3 & 42.9 & 4816 & 57.7 & - \\
\hline No & 4 & 57.1 & 3527 & 42.3 & - \\
\hline Unknown & 4 & - & 186 & - & - \\
\hline $\begin{array}{l}\text { Axillary lymph node status } \\
\text { (invasive cancer) } \mathbb{}\end{array}$ & - & - & - & - & 0.2745 \\
\hline Positive & 2 & 40.00 & 1190 & 20.6 & - \\
\hline Negative & 3 & 60.00 & 4599 & 79.4 & - \\
\hline Unknown & 4 & - & 96 & - & - \\
\hline HIV-status & - & - & - & - & - \\
\hline Positive & 2 & 33.33 & Unknown & - & - \\
\hline Negative & 4 & 66.67 & Unknown & - & - \\
\hline Unknown & 5 & - & Unknown & - & - \\
\hline Total number of cancers & 11 & - & 8529 & - & - \\
\hline
\end{tabular}

Source: Lehman C, Arao R, Sprague B, et al. National performance benchmarks for modern screening digital mammography: Update from the breast cancer surveillance consortium. Radiology. 2017;283(1):49-58. https://doi.org/10.1148/radiol.2016161174

CHBAH, Chris Hani Baragwanath Academic Hospital; RSNA, Radiological Society of North America; HIV, human immunodeficiency virus.

$\dagger, \mathrm{DCIS}$ post-surgical tumour size as measured on pathology specimen, where available $(n=1)$ : intermediate-grade $100 \mathrm{~mm}$; high-grade unknown size; $\$$, Invasive cancer postsurgical tumour size as measured on pathology specimen, where available $(n=5)$. Median $20.00 \mathrm{~mm}$ (interquartile range 9.5-26), mean $18.2 \mathrm{~mm}$ (standard deviation 9.5); benchmark mean $15.9 \mathrm{~mm}(p=0.3043)$. $\$$. Defined as duct carcinoma in situ or invasive cancer $\leq 10$ $\mathrm{mm} ; \boldsymbol{\Phi}$, Refers only to invasive cancers with available nodal pathology results $(n=5)$.

\section{Diagnostic}

Similar audit results were derived for the diagnostic subgroup.

The study population consisted of $93.39 \%(n=693)$ female and $6.61 \%(n=49)$ male patients. The median age was 48 (IQR 40.0-60.0), with a range of 19-91 years. A personal history of breast cancer was recorded in $3.24 \%(n=24)$. The median age of cancer-positive cases was 54.5 (IQR 41.0 $65.0)$, with a minimum of 26 and a maximum of 91 years (Table 3).

Amongst the male patients $(n=49)$, five had abnormal interpretations of their mammograms, consisting of BI-RADS $3(n=2)$, BI-RADS 4c $(n=2)$ and BI-RADS $5(n=1)$ assessments. Two core biopsies were performed, yielding negative results with no detected breast cancer in males.

The presence of a palpable breast mass constituted 55.74\% ( $n=413$ ) of the diagnostic indications (Figure 2). Amongst these patients presenting with a mass, $27.85 \%(n=115)$ of the studies resulted in a diagnosis of cancer, contributing 


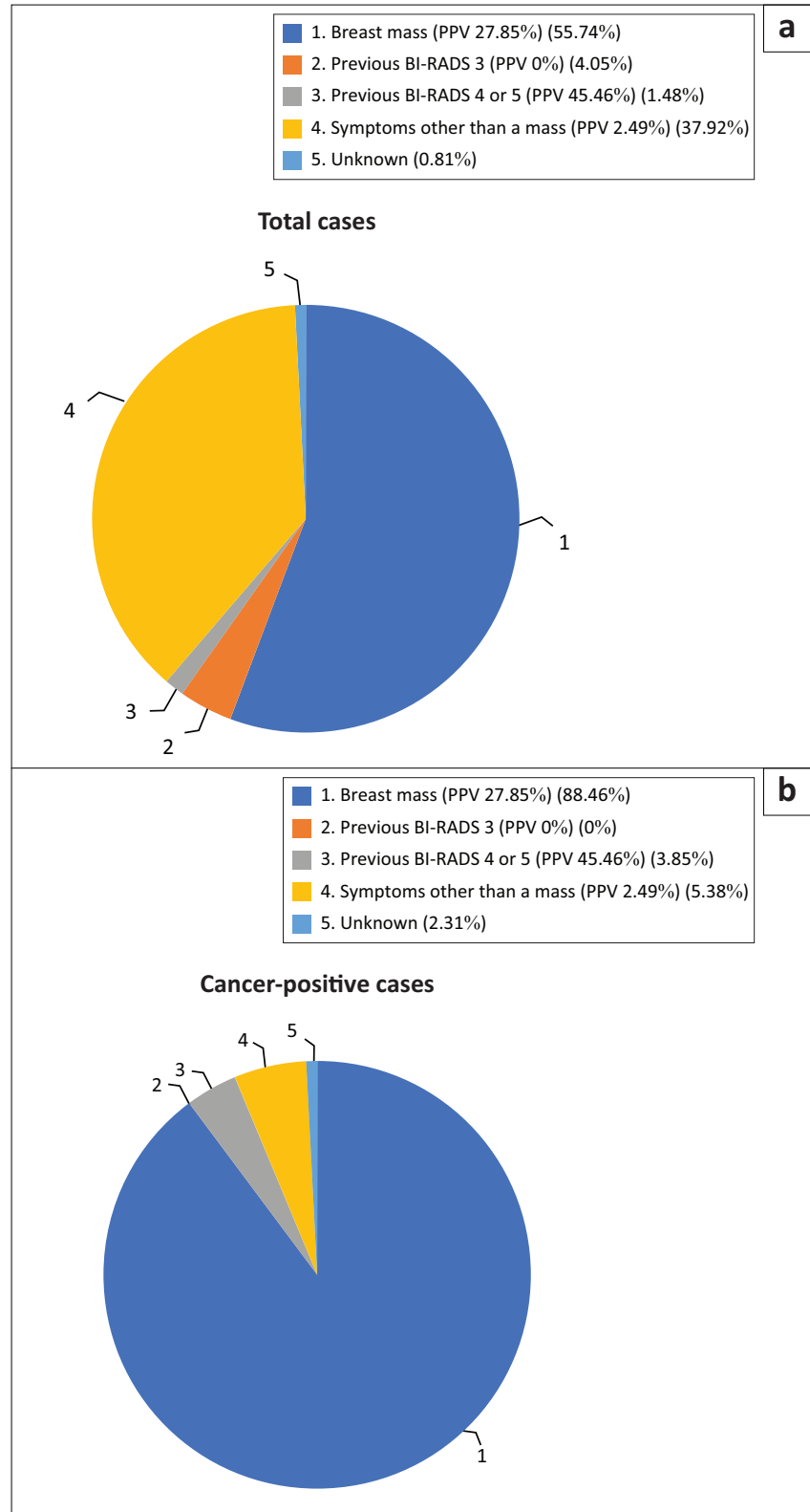

Source: Sprague B, Arao R, Miglioretti D, et al. National performance Benchmarks for modern diagnostic digital mammography: Update from the breast cancer surveillance consortium Radiology. 2017;283(1):59-69. https://doi.org/10.1148/radiol.2017161519

Note: Data in parentheses are positive predictive values (PPVs).

FIGURE 2: Indications for diagnostic mammography: Total examinations and cancer-positive cases.

$88.46 \%$ to the cancer cases detected within the diagnostic subgroup.

The remainder of the indications included: mastalgia $(n=176, \mathrm{PPV}=2.27 \%)$, nipple discharge $(n=36, \mathrm{PPV}=$ $5.56 \%)$, nipple retraction $(n=2, \mathrm{PPV}=0 \%)$, skin changes $(n$ $=9, \mathrm{PPV} 11.11 \%)$, breast abscess $(n=3, \mathrm{PPV}=0 \%)$, gynaecomastia $(n=31$, PPV $=0 \%)$, axillary lymph nodes $(n$ $=4, \mathrm{PPV}=0 \%)$, non-specified breast symptoms $(n=17$, PPV $=0 \%)$, previous BI-RADS $3(n=30, \mathrm{PPV}=0 \%)$ and previous BI-RADS 4 or 5 , where no histology was obtained $(n=11$, $\mathrm{PPV}=45.46 \%)$.

There were 289 (39\%) abnormal interpretations (BI-RADS 3, 4 or 5$)$ and $226(30.50 \%)$ positive studies (BI-RADS 4 or 5). In
TABLE 6: Derived performance measures for diagnostic mammography $(n=741)$.

\begin{tabular}{|c|c|c|c|c|c|}
\hline Measure & $\begin{array}{l}\text { CHBAH } \\
\text { audit value }\end{array}$ & $95 \% \mathrm{Cls}$ & $\begin{array}{l}\text { RSNA- } \\
\text { benchmark } \\
\text { value }\end{array}$ & $95 \% \mathrm{Cl}$ 's & $p$ \\
\hline $\begin{array}{l}\text { Abnormal interpretation } \\
\text { rate (per } 100 \text { studies) }\end{array}$ & 39 & $35.55,42.56$ & 12.6 & $12.5,12.7$ & $<0.0001$ \\
\hline $\begin{array}{l}\text { Number of abnormal } \\
\text { interpretations }\end{array}$ & 289 & - & 50659 & - & - \\
\hline $\begin{array}{l}\text { Total number of } \\
\text { examinations }\end{array}$ & 741 & - & 401548 & - & - \\
\hline $\begin{array}{l}\text { Cancer detection rate } \\
\text { (per } 1000 \text { studies) }\end{array}$ & 175.4 & $149.7,204.4$ & 34.7 & $34.1,35.2$ & $<0.0001$ \\
\hline $\begin{array}{l}\text { Number of cancers } \\
\text { detected }\end{array}$ & 130 & - & 13915 & - & - \\
\hline $\begin{array}{l}\text { Total number of } \\
\text { examinations }\end{array}$ & 741 & - & 401548 & - & - \\
\hline $\mathrm{PPV}_{2}$ & 55.75 & $49.23,62.08$ & 27.5 & $27.1,27.9$ & $<0.0001$ \\
\hline $\begin{array}{l}\text { Number of cancers } \\
\text { detected }\end{array}$ & 126 & - & 13915 & - & - \\
\hline BI-RADS 4, 5 & 226 & - & 50659 & - & - \\
\hline $\mathrm{PPV}_{3}$ & 56.76 & $50.18,63.32$ & 30.4 & $29.9,30.9$ & $<0.0001$ \\
\hline $\begin{array}{l}\text { Number of cancers } \\
\text { detected }\end{array}$ & 126 & - & 10725 & - & - \\
\hline BI-RADS 4, 5 with biopsy & 222 & - & 35275 & - & - \\
\hline
\end{tabular}

Source: Sprague B, Arao R, Miglioretti D, et al. National performance Benchmarks for modern diagnostic digital mammography: Update from the breast cancer surveillance consortium. Radiology. 2017;283(1):59-69. https://doi.org/10.1148/radiol.2017161519

CHBAH, Chris Hani Baragwanath Academic Hospital; RSNA, Radiological Society of North America; $\mathrm{Cl}$, confidence interval; PPV, positive predictive value; BI-RADS, breast-imaging reporting and data system.

$98.23 \%(n=222)$ of the positive studies, tissue diagnosis was obtained within the unit.

The performance measures (Table 6) ${ }^{3}$ revealed an AIR of 39, CDR of 175.4 per 1000 studies $(n=130)$ and a $\mathrm{PPV}_{2}$ and $\mathrm{PPV}_{3}$ of 55.75 and 56.76, respectively. These metrics were all significantly higher than the RSNA-benchmark values $(p<0.0001)$.

The vast majority, $96.15 \%(n=125)$, of the cancers detected were invasive and the remaining $3.85 \%(n=5)$ were DCIS (Table 7). One patient with low-grade DCIS presented with a nipple discharge, whilst the remaining intermediate-grade $(n=3)$ and high-grade $(n=1)$ DCIS lesions were palpable masses. The median tumour size for DCIS lesions on available pathological specimens was 20 mm $(n=2)$. Six patients presented with synchronous bilateral breast cancer $(4.62 \%)$, six with unilateral multicentric cancer $(4.62 \%)$ and seven with multifocal cancer $(5.39 \%)$.

Amongst the invasive cancers with a known pathological tumour size ( $n=58), 82.76 \%$ were greater than $20 \mathrm{~mm}$ with a median tumour length of $31 \mathrm{~mm}$ (IQR 23.0-45.0). Ten cases $(15.87 \%)$ were defined as minimal cancers. In approximately half of the cancer-positive cases $(50.4 \%$, $n=63$ ) the pathological nodal status was available. Of these, $69.84 \%(n=44)$ were nodal-positive and $30.16 \%$ $(n=19)$ nodal-negative. Figure 3 illustrates the comparison of these tumour characteristics with the RSNA-benchmark values, ${ }^{3}$ depicting a larger mean invasive cancer size with a lower percentage of minimal and nodal-negative cancers $(p<0.0001)$. 
TABLE 7: Derived cancer characteristics for diagnostic mammography $(n=130)$.

\begin{tabular}{|c|c|c|c|c|c|}
\hline Characteristic & $\begin{array}{l}\text { CHBAH } \\
\text { audit value }\end{array}$ & $\%$ & $\begin{array}{c}\text { RSNA- } \\
\text { benchmark } \\
\text { value }\end{array}$ & $\%$ & $p$ \\
\hline Cancer type & - & - & - & - & $<0.0001$ \\
\hline Ductal carcinoma in-situ (DCIS) $\dagger$ & 5 & 3.85 & 3329 & 23.9 & - \\
\hline Low-grade & 1 & - & Unknown & - & - \\
\hline Intermediate-grade & 3 & - & Unknown & - & - \\
\hline High-grade & 1 & - & Unknown & - & - \\
\hline Invasive & 125 & 96.15 & 10586 & 76.1 & - \\
\hline Invasive cancer size $(\mathrm{mm}) \$$ & - & - & - & - & $<0.0001$ \\
\hline $1-5$ & 3 & 5.17 & 955 & 9.5 & - \\
\hline $6-10$ & 2 & 3.45 & 1858 & 18.4 & - \\
\hline $11-15$ & 1 & 1.72 & 2049 & 20.3 & - \\
\hline $16-20$ & 4 & 6.9 & 1444 & 14.3 & - \\
\hline$>20$ & 48 & 82.76 & 3767 & 37.4 & - \\
\hline Unknown & 67 & - & 513 & - & - \\
\hline Minimal cancer§ & - & - & - & - & $<0.0001$ \\
\hline Yes & 10 & 15.87 & 6097 & 45.6 & - \\
\hline No & 53 & 84.13 & 7260 & 54.4 & - \\
\hline Unknown & 67 & - & 558 & - & - \\
\hline $\begin{array}{l}\text { Axillary lymph node status } \\
\text { (invasive cancer)ף }\end{array}$ & - & - & - & - & $<0.0001$ \\
\hline Positive & 44 & 69.84 & 3083 & 30.4 & - \\
\hline Negative & 19 & 30.16 & 7074 & 69.6 & - \\
\hline Unknown & 62 & - & 429 & - & - \\
\hline HIV-status & - & - & - & - & - \\
\hline Positive & 34 & 69.91 & Unknown & - & - \\
\hline Negative & 79 & 30.09 & Unknown & - & - \\
\hline Unknown & 17 & - & Unknown & - & - \\
\hline Total number of cancers & 130 & - & 13915 & - & - \\
\hline
\end{tabular}

Source: Sprague B, Arao R, Miglioretti D, et al. National performance Benchmarks for modern diagnostic digital mammography: Update from the breast cancer surveillance consortium. Ragiology. 2017;283(1):59-69. https://doi.org/10.1148/radiol.2017161519

CHBAH, Chris Hani Baragwanath Academic Hospital; RSNA, Radiological Society of North America; HIV, human immunodeficiency virus.

$\dagger$, DCIS post-surgical tumour size as measured on pathology specimen, where available $(n=2)$ low-grade unknown size; intermediate-grade $18 \mathrm{~mm}$; high-grade $22 \mathrm{~mm}$; $\$$, Invasive cancer postlow gul tumour size as meared on pathol surgical tumour size as measured on pathology specimen, where avalable $(n=58)$. median 31 $21.2 \mathrm{~mm}(p<0.0001)$; $\$$, Defined as ductal carcinoma in situ or invasive cancer $\leq 10 \mathrm{~mm}$; $\uparrow$, Refers

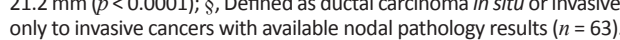

\section{Discussion}

As expected, the data derived from the screening and diagnostic subgroups revealed important differences, illustrating the value of performing separate audits.

\section{Screening}

The comparison of the screening audit to RSNA-benchmark values was limited, as expected from the literature review. This was because of the small number of screening-detected cancers limiting the statistical significance of comparing cancer characteristics. Additionally, the differences in screening practices limited the clinical significance of comparing performance measures. The only parameters that showed a statistically significant difference were a lower recall rate/AIR $(p<0.0001)$, a higher $\operatorname{PPV}_{1}(p<0.0001)$ and a higher CDR $(p=0.0006)$.

The screening audit included patients with a known history of previous breast cancer $(n=277,34.28 \%$ ). The ACR audit guidelines advise for these asymptomatic patients to be regarded as screening investigations. Six (54.55\%) of the 11

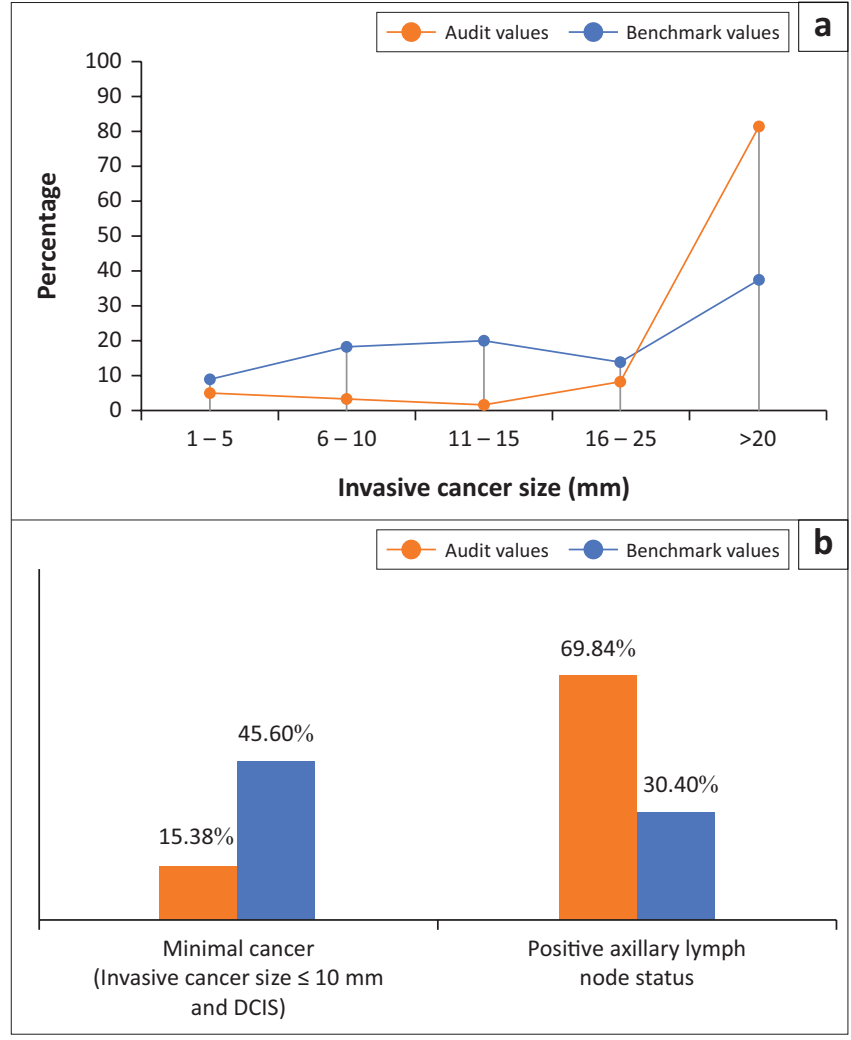

DCIS, ductal carcinoma in situ.

FIGURE 3: Comparative illustration of diagnostic audit values and benchmark values for cancer characteristics based on known pathological cancer size and axillary lymph node status.

cancers detected were from this high-risk subgroup. In the RSNA screening audit, patients with previous breast cancer constituted only $5.1 \%$ of the total patients screened $(n=61628)$ and $15 \%$ of the cancer cases diagnosed $(n=1022) .{ }^{6}$ The majority of patients who received screening mammography at $\mathrm{CHBAH}$ had previously presented with a breast complaint, including breast cancer. Therefore, the screening population at $\mathrm{CHBAH}$ likely represented a high-risk group for the development of breast cancer, impacting the CDR. The exception to this was the month of October (national breast cancer awareness month), during which screening was promoted. Most of the cancers in the subgroup of women with no history of previous breast cancer were detected during this screening-period, likely representing a more accurate estimate of the CDR in women of average risk within our setting.

The difference in recall rate and $P P V_{1}$ can be explained by the difference in screening practices, and is largely influenced by the number of BI-RADS 0 cases. In screening practices where batch reading of mammography is performed, patients are often assessed as BI-RADS 0 and recalled for additional mammography views, tomosynthesis and/or ultrasound. This is standard practice within most facilities in the USA. At $\mathrm{CHBAH}$, immediate reading of screening mammography with tomosynthesis and additional breast ultrasounds are generally performed at the time of first presentation. This is important within a practice serving a large drainage area, where accessibility to breast-imaging units is poor with difficulties in patient recall and follow-up. Consequently, 
there were no patients categorised as BI-RADS 0 and the recall rate included final assessments of BI-RADS 3, 4 and 5. This resulted in a significantly lower recall rate and higher $\mathrm{PPV}_{1}$.

The relative larger size of screen-detected cancers with fewer minimal and nodal-negative invasive cancers, as compared with the RSNA-benchmarks, could be related to the higher frequency of opportunistic screening being performed within the USA. Moreover, the lower PPVs in the RSNA-benchmarks could be attributed to the specific screening environment within the USA (especially regarding self-funding and litigation), where less emphasis is placed on reducing false positive examinations. Despite the comparison of these audit metrics not being statistically significant, the findings are in keeping with what could be expected from the literature review.

The results should, therefore, be interpreted with caution and reflect the differences in screening practices rather than improved performance. This highlights the limitation of comparative audits to international benchmarks. A comparison with follow-up audits in the same unit or units with similar screening practices would be valuable.

The median age of screen-detected cancers was 55 years, which proved to be younger than what was found in the RSNA screening benchmarks. The 40-49 and 50-59 year age groups collectively contributed the majority of cancers detected $(36.36 \%$ and $18.18 \%$, respectively), compared with the RSNA-benchmarks in which most cancers were detected in women over the age of 60 years (56.3\%). In addition, within the subgroup of patients with no personal history of previous breast cancer $(n=5)$, all screen-detected cancers were in the $40-49$ and 50-59 year age groups, (60\% and 40\%, respectively), with a median age of 48 years. This indicates that screening in our setting should be commenced at the age of 40 and would especially benefit women in their 40s and 50s.

The vast majority of patients, in both the screening and diagnostic audits, had low density breasts (type A or B). This could be related to the demographics of the study population, however, would need further investigation.

\section{Diagnostic}

The presence of a palpable breast mass was an important discriminator amongst the reported indications. It was the most common presenting breast complaint and contributed to the majority of cancer cases $(88.46 \%)$ in the diagnostic subgroup. This was similar to what was reported within the RSNA diagnostic audit. On follow-up audits, the results could be subdivided into 'mass' and 'non-mass' categories, with a different set of performance measures and tumour characteristics for each group.

The performance measures within the diagnostic audit revealed higher CDR and PPVs, as compared with the RSNAbenchmarks. This may be attributed to the fact that patients presented with a significantly larger mean tumour length.
The lower percentages of minimal and nodal-negative cancers further reflect an advanced loco-regional stage at presentation.

The reason for the discrepancy between the number of biopsies advised for positive examinations (from which $\mathrm{PPV}_{2}$ is derived) and the number of biopsies performed (from which $\mathrm{PPV}_{3}$ is derived) is not clear. Due to the risk of patients defaulting on follow-up, the department strives to perform immediate image-guided biopsies on all positive imaging examinations.

The marked differences in the performance measures, as well as tumour characteristics compared with the RSNAbenchmarks are likely linked to factors contributing to late presentation of disease within our setting. This could include poor breast cancer awareness and accessibility to healthcare facilities, including the lack of a national mammography screening programme. Chris Hani Baragwanath Academic Hospital is a referral centre with a large component of diagnostic studies. There are also multiple delays in the successful diagnosis and referral of these patients by the referring hospitals, mostly because of various human and other resource constraints. Another consideration may be a more aggressive nature of disease in our population. Further investigation in this regard is needed.

The results highlight the need for the promotion of breast cancer awareness and education to all South African women. Furthermore, providing mammography screening facilities in local clinics would increase adherence to recommended screening guidelines and greatly improve early detection and downstaging of cancers.

\section{Study limitations}

In addition to the previously mentioned limitations of performing comparative audits using international benchmarks, the following points were noted.

\section{General audit limitations:}

- The lack of availability of a national tumour registry precluded the evaluation of metrics, such as falsenegative values, sensitivity and specificity.

- The unit does not have a routine patient self-questionnaire. Patient referral forms were relied upon for clinical data acquisition and, where inadequate, the study was omitted. Patient questionnaires within the unit could have facilitated more accurate clinical data collection.

Additional limiting factors on comparison of the audit results with the benchmark values:

- The audit was performed over 6 months from studies done in 2018, whereas the benchmark articles included studies from 2007 to 2013.

- At CHBAH, the BI-RADS assessment is based on digital tomosynthesis mammography with the addition of 
ultrasound in most cases. The RSNA-benchmark data is based on digital mammography alone.

- Male patients were included in our diagnostic audit, whereas the RSNA-benchmarks were limited to female patients only. This, however, only constituted a small number of abnormal interpretations $(n=5)$, with no contribution to the cancers detected.

- Chris Hani Baragwanath Academic Hospital is an academic referral institution, whereas the data collected for the RSNA-benchmarks were largely from nonacademic, community-based institutions.

\section{Recommendations for future research}

Regular follow-up audits within a facility would be of great value for continuous quality control, especially when a change in practice is implemented. Performance measures could be obtained for each interpreting radiologist. In addition, comparative audits within different South African breast imaging units would provide valuable comparisons of local practices.

We propose a modified recall rate to be used in the auditing of screening practices in South Africa. This should be based on the final BI-RADS assessment after further evaluation with digital breast tomosynthesis and ultrasound is complete, as was done in this study. A final BI-RADS assessment leading to anything other than routine follow-up screening mammography (BI-RADS 3, 4 and 5) should be regarded as an abnormal interpretation and used to calculate the modified recall rate. This would provide a metric distinct from the internationally accepted recall rate, which would be more applicable to many South African screening practices and allow for more relevant comparisons and future research within the country.

The data obtained from these audits could contribute to a breast-imaging database, providing a baseline for the development of benchmarks and recommendations appropriate to the South African setting.

\section{Conclusion}

The study highlights the unique challenges faced by a breastimaging unit within a South African government, tertiary hospital setting. A large proportion of diagnostic mammography is being performed on a population presenting with advanced loco-regional disease, as compared with international, first world benchmarks. It further illustrates the cancer burden within a community where there is a lack of national screening mammography programmes and the additional need for breast cancer awareness.

\section{Acknowledgements}

The authors would like to acknowledge Janike Warnich for her assistance with compiling the tables and figures.

\section{Competing interests}

The authors have declared that no competing interests exist.

\section{Author's contributions}

I.W. was the principal investigator and responsible for the study concept and design, literature review, collection and analysis of data, construction of the database and primary compilation of the manuscript. I.M.V and M.K. were the supervisors and made significant conceptual and editorial contributions to the protocol and final manuscript.

\section{Funding information}

This research received no specific grant from any funding agency in the public, commercial or not-for-profit sectors.

\section{Data availability statement}

Raw data were generated at Chris Hani Baragwanath Academic Hospital. Derived data supporting the findings of this study are available from the corresponding author I.W. on request.

\section{Disclaimer}

The views and opinions expressed in this article are those of the authors and do not necessarily reflect the official policy or position of any affiliated agency of the authors.

\section{References}

1. Bray F, Ferlay J, Soerjomataram I, Siegel R, Torre L, Jemal A. Global cancer statistics 2018: GLOBOCAN estimates of incidence and mortality worldwide for 36 cancers in 185 countries. CA: Cancer J Clin. 2018;68(6):394-424. https://doi.org/10.3322/ caac. 21492

2. Sickles EA, D'Orsi CJ, Bassett LW, et al. ACR BI-RADS $($ Mammography. In: ACR BIRADS ${ }^{\circledR}$ Atlas, Breast imaging reporting and data system. Reston, VA: American College of Radiology, 2013; p. 121-140.

3. Sprague B, Arao R, Miglioretti D, et al. National performance benchmarks for modern diagnostic digital mammography: Update from the breast cancer surveillance consortium. Radiology. 2017;283(1):59-69. https://doi.org/10.1148/ radiol.2017161519

4. Sickles EA, D'Orsi CJ. ACR BI-RADS Follow-up and outcome monitoring. In: ACR BI-RADS Atlas, Breast imaging reporting and data system. Reston, VA: American College of Radiology, 2013; p. 21-31.

5. D'Orsi C, Sickles E. 2017 breast cancer surveillance consortium reports on interpretive performance at screening and diagnostic mammography: Welcome new data, but not as benchmarks for practice. Radiology. 2017;283(1):7-9. https://doi.org/10.1148/radiol.2017170181

6. Maternal \& Child Health - Maternal \& Woman's Health. Breast cancer prevention and control policy [homepage on the Internet]. South African Department of Health; 2017 [cited 2020 Aug 05]. Available from: http://www.health.gov.za/index. php/shortcodes/2015-03-29-10-42-47/2015-04-30-08-18-10/2015-04-30-08-24-27

7. National Cancer Registry. Cancer in South Africa 2016 report [homepage on the
Internet]. National Institute for Communicable Diseases; 2016 [cited 2020 Aug 05]. Available from: http://www.nicd.ac.za/index.php/centres/national-cancerregistry/cancer-statistics/

8. Lehman C, Arao R, Sprague B, et al. National performance benchmarks for modern screening digital mammography: Update from the breast cancer surveillance consortium. Radiology. 2017;283(1):49-58. https://doi.org/10.1148/radiol. 2016161174

9. Froicu M, Mani K, Coughlin B. Satisfaction with same-day-read baseline mammography. J Am Coll Radiol. 2019;16(3):321-326. https://doi.org/10.1016/j. jacr.2018.10.019

10. Lipschitz S. Screening mammography with special reference to guidelines in South Africa. S Afr J Radiol. 2018;22(2):1-7. https://doi.org/10.4102/sajr.v22i2.1370

11. Monticciolo D, Newell M, Hendrick R, et al. Breast cancer screening for average-risk women: Recommendations from the ACR commission on breast imaging. J Am Coll Radiol. 2017;14(9):1137-1143. https://doi.org/10.1016/j.jacr.2017.06.001 
12. Siu A. Screening for breast cancer: U.S. preventive services task force recommendation statement. Ann Intern Med. 2016;164(4):279. https://doi. org/10.7326/M15-2886

13. Rayne S, Schnippel K, Kruger D, Benn CA, Firnhaber C. Delay to diagnosis and breast cancer stage in an urban South African breast clinic. S Afr Med J. 2019;109(3):159-163. https://doi.org/10.7196/SAMJ.2019.v109i3.13283

14. Fast Stats. Mammography use [homepage on the Internet]. Centers for Disease Control and Prevention; 2015 [cited 2020 Aug 05]. Available from: https://www. cdc.gov/nchs/fastats/mammography.htm
15. Broeders $M$, Moss $S$, Nyström L, et al. The impact of mammographic screening on breast cancer mortality in Europe: A review of observational studies. J Med Screen. 2012;19(1_suppl):14-25. https://doi.org/10.1258/jms.2012.012078

16. The Chris Hani Baragwanath Hospital, South Africa [homepage on the Internet]. [cited 2020 Aug 05]. Available from: https://www.chrishanibaragwanathhospital. co.za/

17. Breast Cancer Awareness Month 2019 [homepage on the Internet]. South African Government; 2019 [cited 2020 Aug 05]. Available from: https://www.gov.za/ speeches/breast-cancer-awareness-month-2019 